\title{
Bridging the gap - Fermi-LAT sources at 20-200 MeV
}

\section{Lea Marcotulli ${ }^{a *}$, Chris Karwin $^{a}$, Marco Ajello ${ }^{a}$, Yong Sheng ${ }^{a}$ on behalf of the Fermi Large Area Telescope Collaboration}

${ }^{a}$ Clemson University,

118 Kinard Laboratory of Physics, Clemson, USA

E-mail: lmarcot@g.clemson.edu

The under-explored $\mathrm{MeV}$ band has an extremely rich scientific potential. Awaiting an all-sky MeV mission, it is now the prime time to take full advantage of the capabilities of the Fermi Large Area Telescope to explore this regime. With more than 12 years of the best available dataset (Pass8), we have developed an all-sky analysis to build a sensitive catalog of sources from 20 to $200 \mathrm{MeV}$. This work will allow us to cover the SED peak of the most luminous gamma-ray blazars, fundamental to understand their nature, and possibly discover a whole new population of $\mathrm{MeV}$ sources. Importantly, this program will start bridging the gap between the $\mathrm{MeV}$ and $\mathrm{GeV}$ energy bands, strongly supporting the scientific case for a future all-sky $\mathrm{MeV}$ mission and enhancing the legacy of the Fermi mission. In this talk I will highlight the scientific potential of this project. I will also discuss the difference with respect to the first catalog of low-energy sources (1FLE, Principe et al. 2018).

$37^{\text {th }}$ International Cosmic Ray Conference (ICRC 2021)

July 12 th - 23rd, 2021

Online - Berlin, Germany

\footnotetext{
*Presenter
} 


\section{Introduction}

The last decade has seen an incredible improvement in the sensitivity of both hard X-and $\gamma$-ray telescopes. This advance has led to the detection of hundreds of both Galactic and extra-galactic sources which show a rising hard X-ray spectrum (photon index, $\Gamma_{\mathrm{X}}<1.5$ ) and falling $\gamma$-ray one $\left(\Gamma_{\gamma}>2\right)$, hence likely peaking in the MeV band [1-6].

For example, the most powerful and distant blazars are brightest in the $\mathrm{MeV}$ band [e.g., 7]. Found even beyond $z=5$ [e.g. 8], in general they harbor extremely massive black holes $\left(M_{\mathrm{BH}} \gtrsim 10^{9} \mathrm{M}_{\odot}\right)$. Since their emission is highly beamed, the detection of a single source implies the existence of $2 \Gamma^{2}$ (i.e., $\sim 450$ for a typical bulk Lorentz factor, $\Gamma \sim 15$, [9]) quasars with similar properties, at the same $z$, with jets pointed somewhere else. In other words, the discovery of each new blazar hosting a billion solar mass black hole allows us to constrain the size of the parent population and to quantify the supermassive black hole space density at high- $z$. Importantly, following blazar evolution studies, [9] compared the supermassive black hole number density of radio-quiet (i.e. without jets) sources versus radio-loud (i.e. with jets). The authors found that while the density of radio-quiet AGNs peaks around $z \sim 2$ (when the universe was $\sim 3.5$ billion years old), the radio-loud one peaks much earlier, around $z \sim 4$ (when the universe was only $\sim 1.5$ billion years old, see Figure 1 left panel). This fact challenges our understanding of black hole growth and evolution in the early universe and might point to a connection between jet activity and fast accretion. Detecting these sources in large numbers will take us a step closer to understanding the formation of supermassive black holes in the early universe $[9,10]$.

Similarly, the poorly understood physics of pulsars can be unveiled by studying these sources in the $\mathrm{MeV}$ band [11]. For instance, the Fermi-Large Area Telescope (LAT) has detected 271 pulsars, but only $\sim 40$ are detected at high-significance $(>5 \sigma)<300 \mathrm{MeV}$ and only 4 at $50-100 \mathrm{MeV}$ [12]. The number of pulsars seen at soft $\gamma$-rays $(<50 \mathrm{MeV})$ therefore remains less than 20 [see 13]. The soft $\gamma$-pulsars are found to have high spin values, they are young and very energetic with respect to other detected Fermi-LAT pulsars. At the hard X-rays they usually display a hard power-law spectral index and reach maximum luminosities typically in the $\mathrm{MeV}$ range, implying a peak around 0.1-1 MeV. While some of these 'MeV pulsars' are found to be radio-loud, for the majority of them neither radio nor $\mathrm{GeV}$ emission has been detected. PSR B1509-58 is an example of an 'MeV pulsar' that has been detected by the LAT. With a soft $\gamma$-ray spectrum and a very hard X-ray one (see Figure 1, right panel, [13, 14]), it was also detected by COMPTEL which unveiled its $\mathrm{MeV}$ peak. The high-energy emission of these pulsars can be explained by synchrotron radiation of pairs in the magnetic field. However, the location of production of such pairs is still highly debated [see, e.g. 11]. Only measurement of the SED peaks for a number of these ' $\mathrm{MeV}$ pulsars' can allow us to test different models, understand the location of particle production and pinpoint the physical processes powering them.

The proposed analysis presented in this talk is aimed to develop, for the first time using an all-sky likelihood analysis, a point source catalog in the $20-200 \mathrm{MeV}$ range, bridging the gap between traditional LAT catalogs and the $\mathrm{MeV}$ energy regime. Creating a new census of the $\mathrm{MeV}$ sky is fundamental because it would both enable us to (i) explore the SED peak of known sources (e.g., high-redshift blazars, pulsars, high mass binaries); (ii) detect for the first time sources that, lacking a dedicated low-energy analysis, could have been missed by other LAT catalogs; and (iii) 

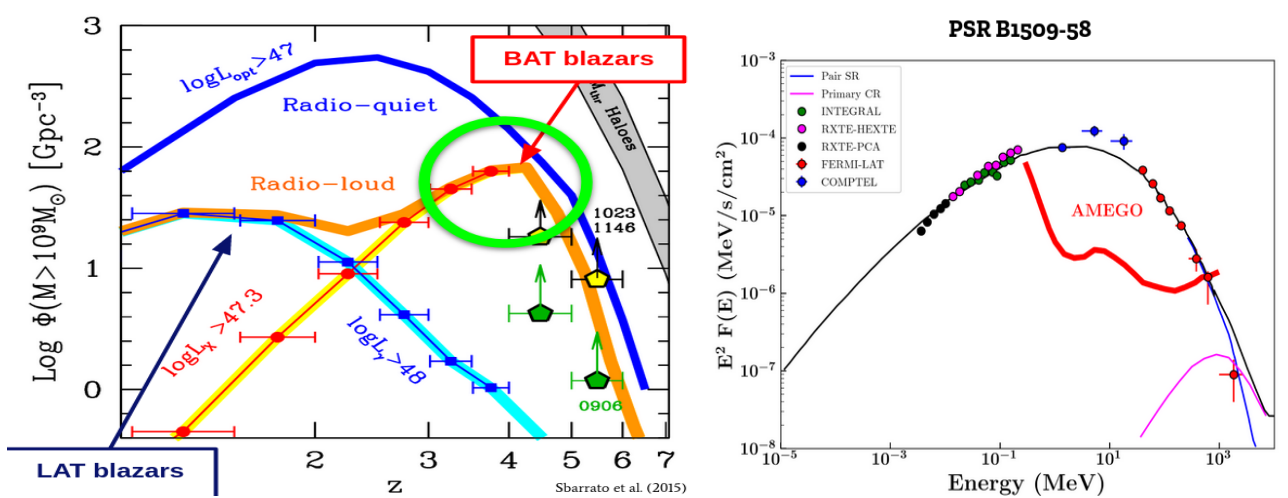

Figure 1: Left: supermassive black hole space density plot, adapted from [9]. Radio-loud sources peak at $z \sim 4-5$, indicating a connection between a jetted phase of the AGN and the fast supermassive black hole growth. Right: high-energy SED of PSR B1509-58, adapted from [11].

provide a 'map' of the $\mathrm{MeV}$ sky in preparation for future $\mathrm{MeV}$ missions.

\section{The Past}

Since the last light of the COMPTEL [15] instrument onboard the Compton Gamma-Ray Observatory, the MeV sky has yet to be explored with good sensitivity. This mission left us with a $\mathrm{MeV}$ catalog comprising 63 objects that emit between 0.75 and $30 \mathrm{MeV}$ (see Figure 2, left panel, $[16,17])$. Among the 32 steady sources detected by COMPTEL, about $1 / 2$ are blazars. Another $1 / 2$ are sources of Galactic origin, such as pulsars, pulsar wind nebulae, and supernova remnants. Lacking an all-sky $\mathrm{MeV}$ instrument, the only chance we have to explore the $\mathrm{MeV}$ regime is to take advantage of the Fermi-LAT low-energy threshold, which allows for the detection of photons down to $20 \mathrm{MeV}$. The majority (>80\%) of the 5065 sources reported in the 4FGL-DR2 catalog [1, 12] has a power-law spectral index $>2$. It is thus clear that most LAT-detected $\gamma$-ray sources emit most of their energy in the $\mathrm{MeV}$ rather than the GeV band. Moreover, the 4FGL-DR2 contains 520 sources detected at a significance level $>5 \sigma$ in the 50-300 MeV energy range. Among those, the majority (86\%) are blazars, $10 \%$ are sources of Galactic origin (pulsars, PWNe, etc.) and few remain unclassified. Only a dedicated analysis in this energy band would enable the detection and precise determination of the spectral properties of these sources and possibly discover more low-energy sources.

Previous work exploiting the LAT capabilities [18] looked for sources between $30-100 \mathrm{MeV}$ using 8.7 years of LAT data and produced the first low-energy catalog of the LAT (1FLE). Their analysis employed a wavelet algorithm (PGWave) and detected 198 sources at $\geq 3 \sigma$. The authors did not use any diffuse emission model to avoid dependence on the imperfections of these models. Of the detected sources, the majority are associated with blazars, $10 \%$ are of Galactic origin and there is also a fraction of $\sim 5 \%$ which does not have any association with the 3FGL [19], hence could potentially be the seed for a new source class to be revealed in the $\mathrm{MeV}$ range.

\section{Analysis and Challenges}

We will use 13 years of LAT data to detect sources in the $20-200 \mathrm{MeV}$ energy band. The detection algorithm is based on a tested implementation of the binned likelihood method, and has 

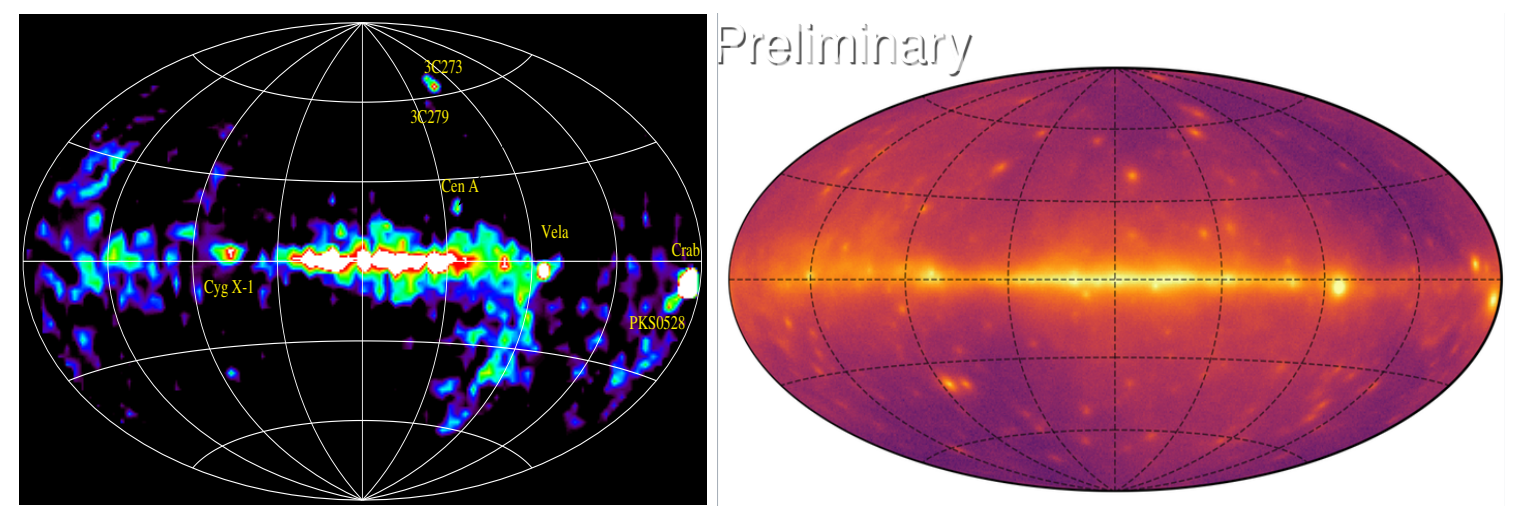

Figure 2: Left: all-sky 1-30 MeV COMPTEL intensity map [15]. A few of the brightest sources are identified, such as powerful blazars (e.g. 3C 273 and 3C 279) and pulsars (e.g. Vela and Crab). Right: preliminary all-sky counts map of PSF3 events between $20-200 \mathrm{MeV}$ using 12 years of LAT data.

the potential to complement and improve over the results of the 1FLE.

Since systematics highly dominate the considered energy range, the analysis has been designed to take them into account and minimize them. Because the LAT angular resolution worsens dramatically with energy ${ }^{1}$, only point spread function (PSF) events that belong to the quartile of the best position reconstruction (PSF3) will be selected [18]. In 12 years of exposure, there are more than 35 million PSF3 events with energy in the $20-200 \mathrm{MeV}$ range. These are contained in the all-sky map shown in Figure 2 (right panel). For comparison, the left panel shows the all-sky map as observed by COMPTEL. Many point-like sources can be seen by eye, indicating that many more will be detected by a dedicated analysis.

We will rely on the Galactic and isotropic diffuse templates ${ }^{2}$ derived for the analysis of the 4FGL. To test the reliability of the sources against systematic uncertainties of the Galactic diffuse emission, we will use alternative Galactic diffuse templates, as is the standard practice [1]. We will also perform end-to-end Monte Carlo simulations to assess the systematics of our analysis. The simulations will contain: the Galactic diffuse emission and isotropic models; the Sun and Moon templates; the templates for spatially extended sources provided in the $4 \mathrm{FGL}^{3}$; and, importantly, the Earth's limb model [19]. Indeed, the spectrum of this component will have to be modeled down to $20 \mathrm{MeV}$, becoming a possible source of systematic uncertainty, and generating spurious sources.

\section{Prospects of Detection}

The prospects of detection in the $\mathrm{MeV}$ band are bright. In fact, using luminosity function studies of the $\mathrm{MeV}$ blazar population (Marcotulli et al. in prep.), we can derive the expected source count distribution (i.e. number of sources as function of flux) of these sources at different $\mathrm{MeV}$ energy bands (see Figure 3, left panel). In the $20-200 \mathrm{MeV}$ band, taking the average LAT sensitivity $\left(S \sim 2 \times 10^{-12} \mathrm{erg} \mathrm{cm}^{-2} \mathrm{~s}^{-1}\right.$ ), one would expect to detect $>1000$ blazars. Therefore constructing a sensitive analysis in this energy range would enable us to produce a catalog that could (i) unveil the

${ }^{1}$ https://www.slac.stanford.edu/exp/glast/groups/canda/lat_Performance.htm

${ }^{2}$ gll_iem_v07.fits and iso_P8R3_SOURCE_V2_PSF3_v1.txt

${ }^{3}$ See 'Extended Source Template Archive'. 


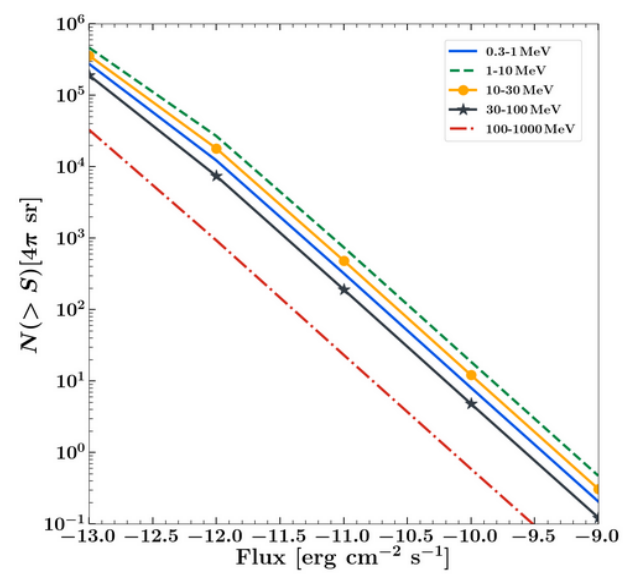

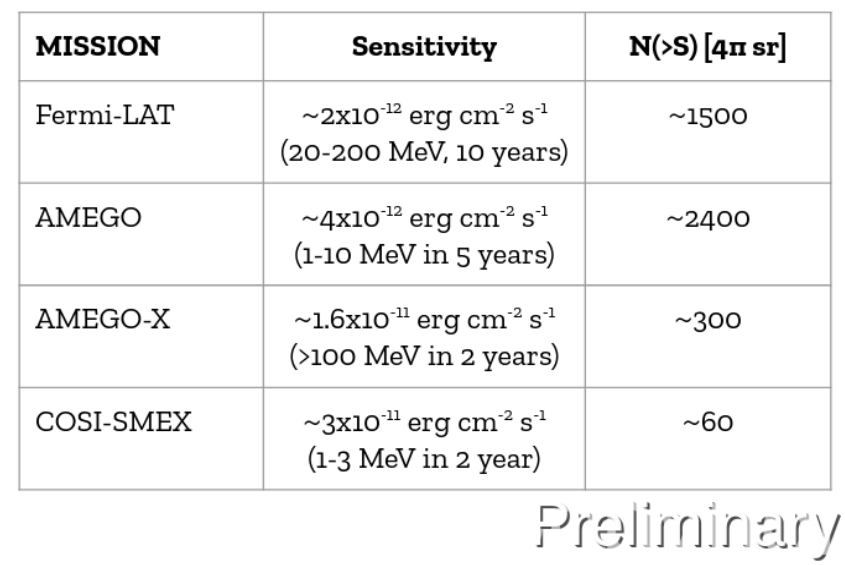

Figure 3: Left: predicted source count distribution of $\mathrm{MeV}$ blazars in different $\mathrm{MeV}$ energy bands (Marcotulli et al. in preparation). Right: blazar counts to be detected by Fermi-LAT and proposed MeV missions based on the predicted sensitivities (Marcotulli et al. in prep).

bulk of this source class and (ii) be used as a map for proposed MeV missions (such as AMEGO [20]) which are expected to detect hundreds to thousands MeV blazars (see Figure 3, right panel).

\section{Summary}

We are developing a new analysis to detect sources in the $20-200 \mathrm{MeV}$ range using 13 years of Fermi-LAT data. This would enable us to explore the SED peak of known sources (e.g., highredshift blazars, pulsars, high mass binaries) and possibly detect for the first time sources that could have been missed by other LAT catalogs. Furthermore, such catalog would provide a map of the $\mathrm{MeV}$ sky in preparation for future $\mathrm{MeV}$ missions (e.g. $\mathrm{AMEGO}^{4}[20]$ or $\mathrm{COSI}^{5}$ ).

\section{Acknowledgments}

The Fermi-LAT Collaboration acknowledges support for LAT development, operation and data analysis from NASA and DOE (United States), CEA/Irfu and IN2P3/CNRS (France), ASI and INFN (Italy), MEXT, KEK, and JAXA (Japan), and the K.A. Wallenberg Foundation, the Swedish Research Council and the National Space Board (Sweden). Science analysis support in the operations phase from INAF (Italy) and CNES (France) is also gratefully acknowledged. This work performed in part under DOE Contract DE-AC02-76SF00515.

\section{References}

[1] S. Abdollahi, F. Acero, M. Ackermann and Ajello, Fermi Large Area Telescope Fourth Source Catalog, 247 (2020) 33 [1902 . 10045].

4https://asd.gsfc.nasa.gov/amego/

5http://cosi.ssl. berkeley.edu 
[2] D. Caprioli, Understanding hadronic gamma-ray emission from supernova remnants, JCC 201 (2011) 026 [1103.2624].

[3] G. Ghisellini, M. Perri, L. Costamante, G. Tagliaferri, T. Sbarrato, S. Campitiello et al., A NuSTAR view of powerful $\gamma$-ray loud blazars, 627 (2019) A72 [1906.02955].

[4] E.V. Gotthelf and S. Bogdanov, NuSTARHard $x$-ray observations of the energetic millisecond pulsars PSR b1821-24, PSR b193721, and PSR j02184232, 845 (2017) 159.

[5] K. Mori, E.V. Gotthelf, C.J. Hailey, B.J. Hord, E. de Oña Wilhelmi, F. Rahoui et al., NuSTAR Hard X-Ray Observation of the Gamma-Ray Binary Candidate HESS J1832-093, 848 (2017) 80 [1710.02118].

[6] K. Oh, M. Koss, C.B. Markwardt, K. Schawinski, W.H. Baumgartner, S.D. Barthelmy et al., The 105-Month Swift-BAT All-sky Hard X-Ray Survey, 235 (2018) 4 [1801.01882].

[7] L. Marcotulli, V. Paliya, M. Ajello, A. Kaur, S. Marchesi, M. Rajagopal et al., NuSTAR Perspective on High-redshift MeV Blazars, 889 (2020) 164 [2001.01956].

[8] H. An and R.W. Romani, X-Ray Constraints on the Spectral Energy Distribution of the $z=$ 5.18 Blazar SDSS J013127.34-032100.1, 904 (2020) 27 [2009.11450].

[9] T. Sbarrato, G. Ghisellini, G. Tagliaferri, L. Foschini, M. Nardini, F. Tavecchio et al., Blazar candidates beyond redshift 4 observed by Swift, 446 (2015) 2483 [1410.0364].

[10] M. Ajello, L. Costamante, R.M. Sambruna, N. Gehrels, J. Chiang, A. Rau et al., The Evolution of Swift/BAT Blazars and the Origin of the MeV Background, 699 (2009) 603 [0905. 0472].

[11] A.K. Harding and C. Kalapotharakos, MeV Pulsars: Modeling Spectra and Polarization, 7th Int. Fermi Symp., Oct., 2017 [1712 . 02406].

[12] J. Ballet, T.H. Burnett, S.W. Digel and B. Lott, Fermi Large Area Telescope Fourth Source Catalog Data Release 2, arXiv e-prints (2020) arXiv:2005.11208 [2005.11208].

[13] L. Kuiper and W. Hermsen, The soft $\gamma$-ray pulsar population: a high-energy overview, 449 (2015) 3827 [1502.06769].

[14] L. Kuiper, W. Hermsen and A. Dekker, The Fermi-LAT detection of magnetar-like pulsar PSR J1846-0258 at high-energy gamma-rays, 475 (2018) 1238 [1709.00899].

[15] V. Schönfelder, R. Diehl, G.G. Lichti, H. Steinle, B.N. Swanenburg, A.J.M. Deerenberg et al., The imaging Compton telescope COMPTEL on the Gamma Ray Observatory., IEEE $\mathbf{3}$ (1984) .

[16] V. Schönfelder, K. Bennett, J.J. Blom, H. Bloemen, W. Collmar, A. Connors et al., The first COMPTEL source catalogue, 143 (2000) 145 [astro-ph/0002366]. 
[17] A. Strong and W. Collmar, COMPTEL Reloaded: a heritage project in MeV astronomy, 90 (2019) 297 [1907.07454].

[18] G. Principe, D. Malyshev, J. Ballet and S. Funk, The first catalog of Fermi-LAT sources below $100 \mathrm{MeV}, 618$ (2018) A22 [1806.10865].

[19] F. Acero, M. Ackermann, M. Ajello, A. Albert and W.B. Atwood, Fermi Large Area Telescope Third Source Catalog, 218 (2015) 23 [1501.02003].

[20] J. McEnery, A. van der Horst, A. Dominguez, A. Moiseev, A.r. Marcowith, A. Harding et al., All-sky Medium Energy Gamma-ray Observatory: Exploring the Extreme Multimessenger Universe, in Bulletin of the American Astronomical Society, vol. 51, p. 245, Sept., 2019 [1907.07558]. 